\title{
The descent of pieces of the Tunguska cosmic body
}

\author{
Olga G. Gladysheva \\ Astrophysical Department of Ioffe Physical-Technical Institute of RAS, St. Petersburg, Russia; Olga.Gladysheva@mail.ioffe.ru
}

Received 23 August 2012; revised 30 September 2012; accepted 14 October 2012

Copyright (C) 2013 Olga G. Gladysheva. This is an open access article distributed under the Creative Commons Attribution License, which permits unrestricted use, distribution, and reproduction in any medium, provided the original work is properly cited.

\begin{abstract}
There is a hypothesis that fragments of a body from outer space fell to Earth as a result of the Tunguska event. During decades of investigations at the site of the disaster, stone or iron pieces from a cosmic body were not found. In spite of this, the number of adherents of this hypothesis is growing. For one thing, it was determined that the formation of swamp craters at the epicentre of the disaster is contempory with the Tunguska event. Secondly, it was proven that the swamp craters are the result of thermokarst processes: their formation is connected with heat exchange in a permafrost zone. Now we can affirm that fragments of the Tunguska cosmic body (as a comet) with a mass of $10^{-3} \leq M \leq 10 \mathrm{~kg}$ reached the Earth's surface, but they did not form craters. This conclusion was made from the isotopes and composition of element in peat deposits as determined by Kolesnikov's group and L'vov.
\end{abstract}

Keywords: Tunguska Cosmic Body; Craters; Organic Component

\section{INTRODUCTION}

The first person to investigate the craters of the Tunguska event was Kulik [1-3]. He essentially believed that the fan-shaped windfall of trees in a circle with a radius of $\sim 30 \mathrm{~km}$ was connected with the impact and explosion of a cosmic object. Kulik [1] understood that this was unique situation, inasmuch as the literature about meteorites, with the exception chronicles, did not contain a description of windfall resulting from a meteorite. This version was refuted in the fifties, when the area of the epicenter of the explosion was investigated by airplane. A crater corresponding to an explosion with a power of $10^{20}-10^{23} \mathrm{erg} / \mathrm{s}$ could not be found [4].

Reaching the epicenter of destruction of the Tunguska cosmic body (TCB), Kulik [1] noted that swamps at the windfall center were studded with dozens of lately formed flat craters which had a diameter ranging from several to dozens of meters and a depth of a few meters. According to Kulik [2], these craters were formed by the fall of meteorite fragments with a mass of $\mathrm{M}_{\mathrm{vl}} \sim \mathrm{n} \times 10^{4} \mathrm{~kg}$. They did not form deep craters but broke through the peat to the permafrost, to a depth of $\sim 0.5 \mathrm{~m}$ at this place.

As a result of field investigations in 1929-1930, it was discovered that swamps of the central zone of the windfall have signs of increasing level of water. It is necessary to remark that the epicenter of the Tunguska disaster hits the permafrost zone, which spreads across the entire northern part of Siberia and has a boundary several hundred kilometers south of the epicenter. Holes bored by Kulik [3] showed that under the surface $(0-2 \mathrm{~m})$ peat layer there are frozen heterogeneous argillaceous deposits up to a depth of $\sim 25 \mathrm{~m}$, below which water-bearing sands are situated. Kulik [2] assumed that meteorite craters served as artesian wells after separate pieces of the meteorite having a mass of $\mathrm{M}_{\mathrm{vm}} \sim \mathrm{n} \times 10^{5} \mathrm{~kg}$ broken through the permafrost and reached a water-bearing horizon. The depth of terminal intrusion of meteorite fragments was determined to be $25-50 \mathrm{~m} \mathrm{[3]}$.

After intensive investigations of the central zone, craters formed by meteorite fragments were not found [5, 6]. Nevertheless, a repeat attempt to search for large TCB fragments in swamp craters was made in 2009-2010 [7]. They looked for comet matter and assumed that this is transparent ice which was found in crater rims.

The aim of this work was to determine the connection between swamp craters and fragments of the TCB. Moreover, it was to determine the size of the TCB's fragments based on isotope and element composition of the substance which reached the ground.

\section{METEORITE FRAGMENTS}

From time to time, someone comes up with the suggestion that meteorite fragments could not be found because the explosion took place over the swamps and the fragments sank. This is, without doubt, a false conjecture. To assert that the entire central area of the disaster site is 
covered with dozens of craters, Kulik did not specify that the craters are located only within swamps, whereas the greater part of the surface of the windfall is rocky (Figure 1).

Sizes of meteorite fragments which, according to $\mathrm{Ku}-$ lik, have a mass of $\mathrm{M}_{\mathrm{vl}}$ and $\mathrm{M}_{\mathrm{vm}}$ are $>1.5$ and $>3.0 \mathrm{~m}$, correspondingly. It is impossible to miss such large objects if they lay on crags. The size of an impact crater has to be comparable with the object's size and represents a deep hole with a diameter of $2-4 \mathrm{~m}$, whereas the size of an explosive crater has to be considerably greater than the object and has a diameter of $>10 \mathrm{~m}$; these craters have to be surrounded by banks. However, careful search and investigation of cavities showed that numerous hollows have no relation to the fall of fragments of the TCB $[5,6]$.

\section{CRATERS (ROUNDED DEPRESSION) OF SWAMPS}

There is reason to believe that numerous craters (rounded depressions) which were found at swamps close to the epicenter started to form after the Tunguska disaster. According to testimony of inhabitants though this place was waterlogged before the disaster trees grew here

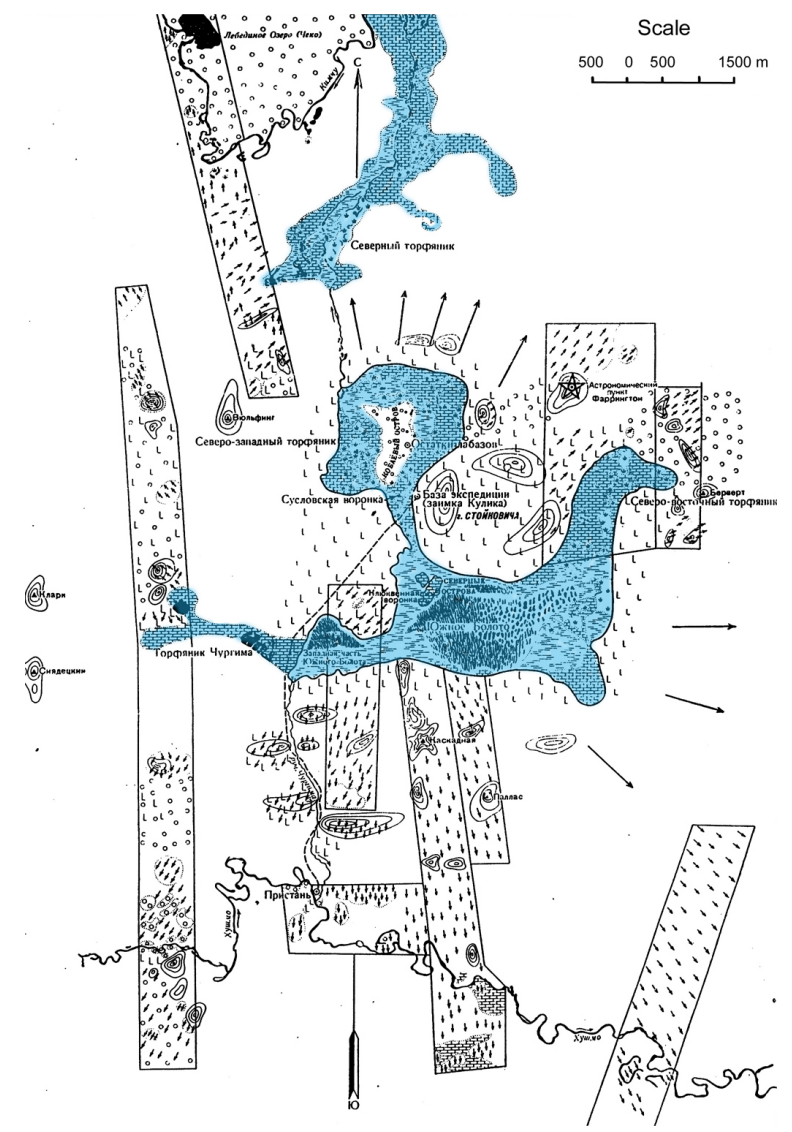

Figure 1. The epicenter of destruction of the TCB (Krinov, 1949). The waterlogged surface is overshadowed. and reindeer pastured. It was determined that depressions are intensively overgrown with sphagnum, the age of which did not exceed the time elapsed from the moment of the disaster [2].

Careful investigations of swamps and rounded depressions were carried out from 1927 to the 1960's [1-3,811]. It was determined that there is no clear connection between the appearance of rounded depressions and the Tunguska event [5-11]. At the same time, an exact connection was found between craters and thermokarst processes, which represents a lowering of surface as a result of irregular melting of underground ice. The necessary conditions for progress of thermokarst processes are a follows [10]: 1) corresponding climatic conditions; 2) a sizable surface of peat $\left(10-15 \mathrm{~m}^{2}\right)$ free from moss; 3$)$ surface inflow of water. During the disaster, trees in swamps were felled by explosion waves. The rhizome system of trees was superficial due to permafrost, and that is why considerable parts of moss sods could be move away with roots [10]. Partial temporary underflooding of a swamp zone after the disaster and global warming, which promoted an intensification of thermokarst processes in other Siberian regions, led to formation of craters.

In the Suslov crater excavated by Kulik several lying trunks was found and at the bottom a tree stump broken close to the root was discovered. This stump was located in a natural position, and its roots normally stretched along the oozy bottom of the crater. This is the evidence of the integrity of the crater bottom [12]. Moreover, during investigations of the South Swamp, 150 holes were bored and 1200 peat columns were drawn out and examined. Anomalous interleaving of peat and soil was not found anywhere in samples [9]. This also speaks against the fall of meteorite fragments in swamps.

\section{COMET SUBSTANCE}

It is necessary to remark that the surface of up-river peat is a unique natural "archive" of matter that falls from the atmosphere. According to [13], the water-mineral food of moss in peat bogs is exceptionally supplied by aerosols, and that is why the chemical composition and pollution of subsoil waters do not influence the moss food. Moreover, peat sediments make possible an exact reading of the peat level, which in 1908 was the surface of the moss sod and where fallen matter was accumulated [14], especially since the level of the peat sediment of 1908 in the epicenter was well-stratified by increased ashing due to the total burn of the moss.

L'vov [13] discovered that peat strata that correspond to the period of the Tunguska event contain "ancient" carbon (depleted ${ }^{14} \mathrm{C}$ ) in a fair quantity. In addition to the "ancient" carbon, isotopic analyses of peat sediments showed that peat levels located at a short distance from the time of the catastrophe are characterized by an ano- 
malous variation of quantities of both ${ }^{13} \mathrm{C}$ and $\mathrm{D}$, enrichment in the isotopic composition of carbon, and depletion in deuterium $[15,16]$. The depletion in radiocarbon $\left({ }^{14} \mathrm{C}\right)$ and enrichment in the $\mathrm{Ir}$ (iridium) in peat sediments corresponding to the time of the disaster (radiocarbon and Ir are two independent indicators of extraterrestrial material.) was confirmed by Rasmussen et al. [17]. Moreover, in these sediments there were found anomalies of elements of the platinum group and rare-earth elements [18], which also are indicators of extra-terrestrial material. Therefore, we can conclude that material of the TCB reached the Earth's surface near the epicenter, and it was a comet substance [19].

The fact that the TCB's substance reached ground and was deposited there means that this comet substance contains a part of more complicated compounds than $\mathrm{CO}_{2}$, $\mathrm{CH}_{4}$, or $\mathrm{CO}$, gases under conditions of Earth. Comparing the allocation of aforesaid isotopic and elements anomalies with the nitrogen anomalies, we find an essential difference. The maximum of the nitrogen isotope variation falls on the permafrost boundary of 1908 [20]. In summer 1908 the permafrost level was at a depth of $\sim 0.5 \mathrm{~m}$. Nitrogen oxides were presumably washed out of the atmosphere by rain and, taking into account the porosity of peat, flowed down with rainwater to the permafrost level. Therefore, we can state that the TCB's material, comet fragments of which left traces on surface sediments in 1908, was either solid or sufficiently viscous at temperatures on Earth.

It is noteworthy that ${ }^{13} \mathrm{C}$ concentrations exceed the background level in the peat sediments that are located in the higher "catastrophe" levels and in the underlying strata [16]. In the underlying strata, ${ }^{13} \mathrm{C}$ was brought either under the influence of shock waves of explosions or as a result of the partial washing of the substance. However, in the overlying deposits carbon came as a result of assimilation by plants. The conclusion is that the comet organics were consumed by plants based on the following. Peat samples containing heavy carbon were processed by a benzol-methanol mixture within 24 hours to remove any soluble compounds (tar, rosin, lipids...). As a result, the isotopic composition of the sample became heavier, and therefore, heavy carbon was located in the cellulose molecules. The peat stratum where isotopic variations were found is asserted to have grown $>13$ years [15]. Thus, we came to the conclusion that during these years organic substances of the comet body gradually oxidised under the influence of bacteria and solar/earth radiation. The organic components of this comet substance broke down into $\mathrm{CO}_{2}$ and $\mathrm{H}_{2} \mathrm{O}$ and were further assimilated by growing moss.

Therefore, we came to the conclusion that fragments of the TCB (as comet) reached the swamp surface, and we can affirm that comet substance carried interplanetary organic material to the Earth's surface.

\section{COMET FRAGMENTS}

An investigation of the peat sediments at the place of the Tunguska disaster showed that fall out of extraterrestrial substance occurred extremely irregularly, as it is clearly shown in separate areas $[13,16]$. For example, a sample taken $15 \mathrm{~km}$ north of the epicenter in the valley of the Maleshko brook contains 10\% "ancient" carbon. Samples from the peat bog Bublik $(6 \mathrm{~km}$ to the northwest of the epicenter) was richest in the matter of the Tunguska body, in which cosmic carbon was $\sim 40 \%$ of the mass of carbon in vegetable remains. By contrast, in the samples from the North peat bog $(1.5 \mathrm{~km}$ northeast of the epicenter) and Tsvetkovsky peat bog $(60 \mathrm{~km}$ from the epicenter near the Vanavara settlement) radiocarbon was within the norm. On the other hand, spottiness was noted within every peat bog. As a rule, only one of several samples had a considerable amount of "ancient" carbon [13]. Therefore, we can conclude that cosmic substance fell as separate fragments.

Based on the samples under investigation (from $2 \times$ $10^{-5}$ to $1 \times 10^{-1} \mathrm{~kg}$ ), which include cosmic substances, along with the fact that peat stratum where isotopic variations were discovered had a thickness of $\sim 5 \mathrm{~cm}$ [15], we could approximately determine the mass of the fragments of the TCB, falling on the earth's surface as $\geq 1 \times$ $10^{-3} \mathrm{~kg}$. An upper limit of the fragment's mass could be roundly estimated as $1-10 \mathrm{~kg}$, inasmuch as larger comet fragments falling from the height of the explosion $(\sim 6$ $\mathrm{km})$ probably must to form craters. Nevertheless, the question about the connection between large $(>10 \mathrm{~kg})$ comet fragments of the TCB with swamp craters needs careful investigation.

In is necessary to remark that, according to Kulik [2], winter frozen and permafrost at the epicenter completely close up to New Year's Day in the presence of a blanket of snow $>0.5 \mathrm{~m}$ and a temperature $<-50^{\circ} \mathrm{C}$. Ice lenses were formed as a result of deformation of frozen peat and spreading under its soil [8]. Thus, it is unlikely that transparent ice which was found in a crater [7] has some relation to the TCB.

\section{ACKNOWLEDGEMENTS}

I am grateful to B.F. Bidyukov, S.V. Krivyakov, V.V. Kopeikin and V.A. Romejko for useful discussions. This work was sponsored by the 22 Program of the Presidium of the Russian Academy of Sciences.

\section{REFERENCES}

[1] Kulik, L.A. (1927) To the question about a place of the Tunguska meteorite fall. Doklady Akademii Nauk SSSR, 399-402.

[2] Kulik, L.A. (1933) To 25-anniversary of the Tunguska 
meteorite. Mirovedenie, 2, 63-66.

[3] Kulik, L.A. (1939) Data about the Tunguska meteorite to 1939. DAS SSSR, 22, 520-524.

[4] Florensky, K.P. (1955) Some impressions about modern state of the region of fall of the Tunguska meteorite 1908 . Meteoritika, 12, 62-71.

[5] Florensky, K.P. (1963) Preliminary result of the Tunguska meteorite complex expedition. Meteoritika, 23, 3-29.

[6] Kuvshinnikov, V.M. and Kolobkova, G.P. (1963) To the questions about a falling mass of the Tunguska meteorite at the Lakura mountain ridge's region. In: Plekhanov, G.F. Ed., The Problem of the Tunguska Meteorite, Izdat Tomsk University, Tomsk, 159-162.

[7] Alekseev, V.A., Alekseeva, N.G. and Kopeikin, V.V. (2011) Results of georadar and hydrogen studies of Tunguska meteorite impact crater in 2009-2010. Atmospheric and Oceanic Optics Journal, 24, 1105-1107.

[8] Shumilova, L.V. (1963) Sketch about the environment at the region of fall of the Tunguska meteorite. In: Plekhanov, G.F., Ed., The Problem of the Tunguska Meteorite, Izdat Tomsk University, Tomsk, 22-33.

[9] L'vov, Y.A., Lagutskaya, L.I., Ivanova, G.M., et al. (1963) Swamps at the region of the fall of the Tunguska meteorite. In: Plekhanov, G.F., Ed., The Problem of the Tunguska Meteorite, Izdat Tomsk University, Tomsk, 34-47.

[10] L'vov, Y.A. and Ivanova, G.M. (1963) Reduced (thermokarst) depression of large bumpy peat bog at the region of fall of the Tunguska meteorite. In: Plekhanov, G.F., Ed., The Problem of the Tunguska Meteorite, Izdat Tomsk University, Tomsk, 48-58.

[11] Kovalevsky, A.F., Fast, V.G., Ikonnikova, G.M. and Nekrasova, L.N. (1963) Magnetic investigations at the region of fall of the Tunguska meteorite. In: Plekhanov, G.F., Ed., The Problem of the Tunguska Meteorite, Izdat Tomsk University, Tomsk, 113-123.

[12] Krinov, E.L. (1949) The Tunguska meteorite. Izddat.
[13] L'vov, Y.A. (1984) Carbon in the structure of the Tunguska meteorite. In: Dolgov, Y.A., Ed., Meteorite Investigations in Siberia (Tunguska Phenomena Is 75 years), Nauka, Novosibirsk, 83-87.

[14] L'vov, Y.A. (1967) About presence of extraterrestrial substance in peat. In: Tronov, M.V., Ed., The Problem of the Tunguska Meteorite 2, Izdat Tomsk University, Tomsk, 140-144.

[15] Kolesnikov, E.M. (1982) Isotope H and C anomalies in peat at the place of fall the Tunguska meteorite. Doklady Akademii Nauk SSSR, 266, 993-995.

[16] Kolesnikov, E.M., Boettger, T. and Kolesnokova, N.V. (1999) Finding of probable Tunguska Cosmic Body material: Isotopic anomalies of carbon and hydrogen in peat. Planetary and Space Science, 47, 905-916. doi:10.1016/S0032-0633(99)00006-9

[17] Rasmussen, K.L., Olsen, H.J.F., Gwozdz, R. and Kolesnikov, E.M. (1999) Evidence for a very high carbon/iridium-ratio in the Tunguska impactor. Meteoritics and Planetary Science, 34, 891-895. doi:10.1111/j.1945-5100.1999.tb01407.x

[18] Hou, Q.L., Kolesnikov, E.M., Xie, L.W., et al. (2000) Discovery of probable Tunguska cosmic body material: Anomalies of platinum group elements and rare-earth elements in peat near the explosion site (1908). Planetary and Space Science, 48, 1447-1455. doi:10.1016/S0032-0633(00)00089-1

[19] Kolesnikov, E.M., Hou, Q.L., Xie, L.W. and Kolesnikova, N.V. (2005) Finding of probable Tunguska cosmic body material: Anomalies in platinum group elements in peat from the explosion area. Astronomy and Astrophysics, 24, 101-111. doi:10.1080/10556790500085678

[20] Kolesnikov, E.M., Kolesnikova, N.V. and Boettger, T. (1998) Isotopic anomaly in peat nitrogen is a probable trace of acid rains caused by 1908 Tunguska bolide. Planetary and Space Science, 46, 163-167. doi:10.1016/S0032-0633(97)00190-6 